\title{
HIGH-FREQUENCY SUSCEPTIBILITIES OF INHOMOGENEOUS MAGNETOELASTIC MEDIUM
}

\author{
V.A. IGNATCIIENKo and M.V. EREMENTChouk \\ L.V. Kirensky Institute of Plysics, 660036, Krasnoyarsk, Russia
}

\begin{abstract}
A response of an inhomogeneous magnetoelastic medium with the zero-mean magnetostriction to high-frequency elastic and electromagnetic fields is studied. It is shown that diagonal susceptibilities have the resonance anomalies in the vicinity of the frequency of the magnetoelastic resonance, while the coefficients of crossing excitations are equal to zero.
\end{abstract}

PACS numbers: $75.80 .+q, 61.43 . E r, 62.30 .+d$

A model of the disorder induced crossing-resonance (DICR) was first considered in Ref. [1]. On the basis of the model the magnetoelastic resonance in inhomogeneous ferromagnets was studied $[2,3]$. In the model the average magnetostriction was assumed to be equal to zero and a coupling between spin and elastic waves arose only due to spatial fluctuations of the magnetostriction parameter. Such situation exists, for example, in the zero-magnetostrictive amorphous ferromagnets [4]. For the model the eigenfrequencies [1-3] and polarization [3] of waves in the DICR vicinity were considered. In the present paper the components of the matrix of susceptibility (i.e. Fourier transforms of averaged Green's functions) of amorphous ferromagnets are studied, which describe the amplitudes and phases of both spin and elastic waves excited. The excitations arise from the action either of the external magnetic fields $h$ or of the elastic forces $f$.

Equations of motion for magnetoelastic media have the following form:

$$
\dot{M}=-g\left[M \times\left(-\frac{\partial U}{\partial M}+\frac{\partial}{\partial x} \frac{\partial U}{\partial(\partial M / \partial x)}\right)\right], \quad G \ddot{u}_{i}=\frac{\partial}{\partial x_{i}} \frac{\partial U}{\partial u_{i j}},
$$

where $M$ is the magnetization vector, $u_{i j}=\frac{1}{2}\left(\partial u_{i} / \partial x_{j}+\partial u_{j} / \partial x_{i}\right)$ is the elastic deformation tensor, $u$ is the displacement vector, $g$ is the gyromagnetic ratio and $G$ is the density of the material.

We take potential energy $U$ in the form corresponding to isotropic medium

$U=\frac{1}{2} \alpha(\nabla M)^{2}-H \cdot M+\frac{1}{2} d_{1} u_{i i}^{2}+\frac{1}{2} d_{2}\left(u_{i j} u_{i j}+u_{i j} u_{j i}\right)+\frac{1}{2} P(x) M_{i} M_{j} u_{i j}$.

IIere the first and second summands are the magnetic subsystem energy ( $\alpha$ is the exchange parameter, $\boldsymbol{H}$ is the magnetic field), third and forth ones describe the elastic subsystem energy ( $d_{1}$ and $d_{2}$ are Lame's coefficients) and the last term introduces the magnetoelastic coupling. 
According to the DICR model we consider the situation when the mean value of the magnetoelastic parameter $P(x)$ equals to zero and it can be written as

$$
P(x)=\gamma \rho(x)
$$

where $\gamma$ is the mean square fluctuation of $P(x)$ and $\rho(x)$ is the centralized $(\langle\rho(x)\rangle=0)$ and normalized $\left(\left\langle\rho^{2}(x)\right\rangle=1\right)$ random function which statistic properties are described by the correlation function $K(\boldsymbol{r})=\langle\rho(\boldsymbol{x}) \rho(\boldsymbol{x}+\boldsymbol{r})\rangle$.

Considering small deviations $m(\boldsymbol{x}, l)$ from the equilibrium direction of the magnetization and using the plane wave expansion we obtain a system of integral equations for $m(k, \omega)$ and $u(k, \omega)$. Solutions of the system are found as the powcr series expansions in $\rho(x)$ which are averaged over an ensemble of the random function.

A response of a medium to the external action is described by the matrix $\hat{\chi}$ of the complex susceptibility. Its diagonal components $\chi_{u u}$ and $\chi_{s s}$ correspond to the response of the subsystem $u$ to the $f$ action and of the subsystem $m$ to the $h$ action, respectively. The nondiagonal components describe the cross excitations.

In all orders of the expansion the nondiagonal components contain the products of odd numbers of the random functions $\rho(x)$. They vanish after averaging with any even distribution function. Therefore, in the DICR case, unlike the homogeneous magnetoelastic resonance, the transformation of the averaged spin waves $\langle m\rangle$ into the averaged elastic waves $\langle u\rangle$ and vice versa does not take place. The averaged wave of a field interacts only with the fluctuation waves of another field. The interaction leads to a strong modification of the diagonal components of the susceptibility

$$
\begin{aligned}
& \chi_{u u}=\frac{\left\langle u_{k}\right\rangle}{f_{k}}=\frac{\Omega_{u}^{2}}{\left(\omega-\mathrm{i} \Gamma_{u}\right)^{2}-\omega_{k}^{2}+Q_{u}(\omega, k)}, \\
& \chi_{s s}=\frac{\left\langle m_{k}\right\rangle}{h_{k}}=\frac{\omega_{M}}{\omega-\mathrm{i} \Gamma_{s}-\epsilon_{k}+Q_{s}(\omega, k)},
\end{aligned}
$$

where $\omega$ is the excitation frequency, $\omega_{k}$ and $\epsilon_{k}$ are the dispersion laws of the elastic and spin waves, respectively, $\Gamma_{u}$ and $\Gamma_{s}$ are the initial damping parameters of the elastic and spin waves, $\omega_{M}=g M, \Omega_{u}$ is the force coefficient. The mass operators $Q_{s}$ and $Q_{u}$ were found in the first nonvanishing order of the perturbation theory

$$
\begin{aligned}
& Q_{u}=\frac{\lambda^{2} \omega_{k}}{2} \int \frac{S\left(k-k_{1}\right)}{\omega-\mathrm{i} \Gamma_{s}-\epsilon_{k_{1}}} \mathrm{~d} k_{1}, \\
& Q_{s}=\frac{\lambda^{2} v^{2}}{4 \omega_{k}} \int \frac{\left(k_{1}^{2}+k_{1 z}^{2}\right) S\left(k-k_{1}\right)}{\left(\omega-\mathrm{i} \Gamma_{u}\right)^{2}-\omega_{k_{1}}^{2}} \mathrm{~d} k_{1},
\end{aligned}
$$

where $\lambda^{2}=\gamma^{2} M_{0}^{2} \omega_{M} \omega_{k} /\left(G v^{2}\right), S(k)$ is the Fourier transform of $K(r)$.

The integrals (5) with the correlation function $K(r)=\exp \left(-k_{\mathrm{c}} r\right)$, where $k_{\mathrm{c}}^{-1}$ is the correlation radius of the inhomogeneities, were calculated earlier when dispersion laws had been analysed (see Eqs. (10) and (11) in Ref. [2]). Neglecting the quadratic effects in the damping we can rewrite Eqs. (4) in the following form:

$$
\chi_{u u}=\Omega_{u} \frac{\omega-\epsilon_{k}-\mathrm{i} \Gamma_{s}^{*}}{D_{u}(\omega, k)}, \quad \chi_{s s}=\omega_{M} \frac{\omega-\omega_{k}-\mathrm{i} \Gamma_{u}^{*}}{D_{s}(\omega, k)} .
$$


IIere

$$
\begin{aligned}
& D_{u}=\left(\omega-\omega_{k}-\mathrm{i} \Gamma_{u}\right)\left(\omega-\epsilon_{k}-\mathrm{i} \Gamma_{s}^{*}\right)-\lambda^{2} / 4, \\
& D_{s}=\left(\omega-\epsilon_{k}-\mathrm{i} \Gamma_{s}\right)\left(\omega-\omega_{k}-\mathrm{i} \Gamma_{u}^{*}\right)-\lambda^{2} / 4
\end{aligned}
$$

and the eflective damping parameters $\Gamma_{u}^{*}$ and $\Gamma_{s}^{*}$ are the sums of the initial ones and the dampings caused by the waves scattering on the inhomogeneities

$$
\Gamma_{u}^{*} \approx \Gamma_{u}+v k_{c}, \quad \Gamma_{s}^{*} \approx \Gamma_{s}+2 k_{\mathrm{c}} \sqrt{\alpha \omega_{M}\left(\omega-\omega_{0}\right)} .
$$

The main anomaly of the diagonal components (6) of the susceptibility in the case of the disorder induced magnetoelastic resonance is that their denominators $D_{u}$ and $D_{s}$ are different while in homogeneous media both $\chi_{u u}$ and $\chi_{s s}$ have the common denominator. The difference between $D_{u}$ and $D_{s}$ results from the different additions to the initial dampings in (8) which are proportional to both $k_{\mathrm{c}}$ and the velocity of the corresponding wave. A difference between the elastic and spin waves velocities leads to a stronger increase of the spin subsystem damping (which depends on $\Gamma_{u}^{*}$ ) than in the elastic one.

An analysis of the equations $D_{u}=0$ and $D_{s}=0$ for the eigenfrequencies which follow from Eqs. (7) shows that the conditions of degeneration removal and the gap $\Delta \omega$ appcarance at the point of the crossing resonance $k=k_{\mathrm{r}}$ are different for the elastic and spin waves spectra. They have, respectively, the forms

$$
\lambda^{2}>\left(\Gamma_{s}^{*}+\Gamma_{u}\right)^{2} \quad \text { and } \quad \lambda^{2}>\left(\Gamma_{s}+\Gamma_{u}^{*}\right)^{2} .
$$

These are the expansions of the corresponding expressions derived in Ref. $[1,2]$ without taking into account the initial dampings $\Gamma_{u}$ and $\Gamma_{s}$. One can see that the inclusion of the initial dampings does not change the conclusion obtained there, the spectrum of the average elastic waves may have the gap while the dispersion curve of the spin waves remains continuous or vice versa.

An analysis of Eqs. (6) shows that the conditions of the two maxima existing in $\chi_{u u}(\omega)$ and $\chi_{s s}(\omega)$ curves are also different. They are defined, respectively, by the expressions

$$
\lambda^{2}>4 \Gamma_{s}^{* 3} /\left(2 \Gamma_{s}^{*}+\Gamma_{u}\right) \quad \text { and } \quad \lambda^{2}>4 \Gamma_{u}^{* 3} /\left(2 \Gamma_{u}^{*}+\Gamma_{s}\right) .
$$

Let us consider now the differences between the susceptibilities of DICR and of the homogeneous crossing resonance. The expressions, corresponding to the homogeneous magnetoelastic resonance can be formally deduced from Eqs. (6), (7), (9) and (10), if we take $\Gamma_{u}^{*}=\Gamma_{u}, \Gamma_{s}^{*}=\Gamma_{s}$ and assume that $\lambda$ is proportional to the homogeneous magnetoelastic coupling parameter. One can see that Eqs. (9) become the same but Eqs. (10) are different.

Let $\Gamma_{s}>\Gamma_{u}$ for definiteness. Let the condition (9) for the gap appearance be fulfilled. If, in addition, the conditions (10) permit the presence of two maxima in both $\chi_{u u}$ and $\chi_{s s}$ curves, then the difference betwcen the resonance frequencies for $\chi_{u u}$ will be smaller than $\Delta \omega$ but the same difference for $\chi_{s s}$ will be greater than $\Delta \omega$. It is possible that the resonance peaks in $\chi_{u u}$ approach each other till they are finally joined, however two maxima in $\chi_{s s}$ can be observed. Let now the condition opposite to (9) be fulfilled and the eigenfrequencies coincide at $k=k_{\mathbf{r}}$. It is evident from Eqs. (10) that there is one maximum in the susceptibility $\chi_{u u}$ but $\chi_{s s}$ still has two maxima. This paradoxical at first glance situation is due to 
the fact that under these conditions the phenomenon of an antyphase resonance manifests itself in the susceptibility of the subsystem, corresponding to the larger damping. The phenomenon was first investigated for the coincidence of NMR and FMR frequencies [5] and considered in a more general case in Ref. [6].

Thus, even for the homogeneous crossing resonance there is no one-to-one correspondence between the values of eigenfrequencies and the locations of the susceptibility maxima. For the disorder induced magnetoelastic resonance the relation between the maxima locations and the values of eigenfrequencies becomes more complicated because it involves a larger number of parameters. We restrict our considerations here to the case when there is no gap in the spectra of both spin and elastic waves, i.e. the conditions opposite to (9) are fulfilled; and besides, we assume $\Gamma_{s} \gg \Gamma_{u}$. The estimations show that such relationships between the parameters are characteristic of the amorphous zero-mean magnetostrictive alloys. In this case $\chi_{u u}$ and $\chi_{s s}$ have the following form:

$$
\begin{aligned}
& \frac{2}{\Omega_{u}} \chi_{u u} \approx \frac{(1+\sigma) \xi}{\left(\omega-\omega_{r}\right)^{2}+\xi^{2}}, \\
& \frac{2}{\omega_{M}} \chi_{s s} \approx \frac{\left(1+\sigma_{1}\right) \xi_{+}}{\left(\omega-\omega_{r}\right)^{2}+\xi_{+}^{2}}-\sigma_{1} \frac{\xi_{-}}{\left(\omega-\omega_{r}\right)^{2}+\xi_{-}^{2}},
\end{aligned}
$$

where $\xi=\Gamma_{u}+\sigma, \xi_{+}=\Gamma_{s}-\sigma_{1}, \xi_{-}=\Gamma_{u}^{*}+\sigma_{1}, \sigma=\lambda^{2} / 4\left(\Gamma_{s}^{*}-\Gamma_{u}\right), \sigma_{1}=$ $\lambda^{2} / 4\left(\Gamma_{s}-\Gamma_{u}^{*}\right)$.

As there is only one resonance peak in $\chi_{u u}$, the magnetic susceptibility $\chi_{s s}$ has the structure typical of the antyphase resonance: the high and wide peak of the magnetic resonance absorption and the weak and narrow peak of the elastic resonance irradiation. The peaks have the same resonance frequencies $\omega_{\mathrm{r}}$, but they are opposite in sign. As a result, the curve $\chi_{s s}(\omega)$ has two maxima whose locations do not correspond to any resonance frequencies.

It is seen from Eqs. (11) and (12) that experimental investigation of the disorder induced magnetoelastic resonance could allow measurements of the main characteristics of the magnetostriction inhomogeneities, i.e. the mean square fluctuation $\gamma$ and correlation radius $k_{\mathrm{c}}^{-1}$.

\section{References}

[1] V.A. Ignatchenko, L.I. Deich, Phys. Rev. B 50, 16364 (1994).

[2] L.I. Deich, V.A. Ignatchenko, Zh. Eksp. Teor. Fiz. 107, 842 (1995).

[3] L.I. Deich, M.V. Erementchouk, V.A. Ignatchenko, Zh. Eksp. Teor. Fiz. 109, 1370 (1996).

[4] A.J. Hernando, J. Phys., Condens. Malter 2, 1885 (1990).

[5] V.A. Ignatchenko, V.I. Tsifrinovich, Zh. Eksp. Teor. Fiz. 131, 75 (1975).

[6] V.A. Ignatchenko, V.I. Tsifrinovich, Usp. Fiz. Nauk 131, 75 (1981). 